Supporting Information

\title{
Efficient Removal and Recovery of Uranium by a Layered Organic-Inorganic Hybrid Thiostannate
}

Mei-Ling Feng, ${ }^{1,2}$ Debajit Sarma, ${ }^{2}$ Xing-Hui Qi, ${ }^{1}$ Ke-Zhao Du, ${ }^{1}$ Xiao-Ying Huang, ${ }^{1}$ and Mercouri G.

Kanatzidis $^{*, 2}$

${ }^{1}$ State Key Laboratory of Structural Chemistry, Fujian Institute of Research on the Structure of Matter, Chinese Academy of Sciences, Fuzhou, Fujian 350002, P. R. China.

${ }^{2}$ Department of Chemistry, Northwestern University, 2145 Sheridan Road, Evanston, Illinois 60208, United States

E-mail: m-kanatzidis@northwestern.edu 
Table S1. The date for concentration of uranium $\left(C_{t}\right)$ and the relative amount of $\mathrm{U}$ removed $(R)$ at the different time in kinetic experiments.

\begin{tabular}{lll}
\hline time (minutes) & $C_{t}(\mathrm{ppb})$ & $R(\%)$ \\
\hline 0 & 1021.74 & 0 \\
3 & 500.97 & 50.96 \\
6 & 463.69 & 54.56 \\
15 & 390.58 & 61.77 \\
30 & 350.12 & 65.73 \\
60 & 200.90 & 80.33 \\
120 & 168.29 & 83.52 \\
240 & 107.48 & 89.48 \\
1200 & 85.96 & 91.58 \\
\hline
\end{tabular}

Table S2. pH dependent $\mathrm{UO}_{2}{ }^{2+}$ ion-exchange results.

\begin{tabular}{|c|c|c|c|c|c|}
\hline \multirow[t]{2}{*}{ Initial $\mathrm{pH}$} & \multirow[t]{2}{*}{ Final $\mathrm{pH}$} & \multicolumn{2}{|c|}{ U concentration (ppb) } & \multirow[t]{2}{*}{$K_{\mathrm{d}}(\mathrm{mL} / \mathrm{g})$} & \multirow{2}{*}{$\begin{array}{l}\text { Relative amount of } \mathrm{U} \\
\text { removed (\%) }\end{array}$} \\
\hline & & $C_{0}$ & $C_{f}$ & & \\
\hline 2.1 & 2.1 & 3073 & 2952 & 40.85 & 3.90 \\
\hline 3.8 & 5.1 & 447.5 & 30.0 & $1.39 \times 10^{4}$ & 93.28 \\
\hline 5.8 & 7.0 & 1775 & 64.8 & $2.64 \times 10^{4}$ & 96.35 \\
\hline 6.5 & 7.7 & 1088 & 76.4 & $1.32 \times 10^{4}$ & 92.97 \\
\hline 7.3 & 7.4 & 176.4 & 42.2 & $3.17 \times 10^{3}$ & 76.07 \\
\hline 8.4 & 7.9 & 277.8 & 84.2 & $2.29 \times 10^{3}$ & 69.69 \\
\hline 9.8 & 7.6 & 3580 & 2570 & $3.93 \times 10^{2}$ & 28.21 \\
\hline 10.4 & 8.3 & 2908 & 2288 & $2.71 \times 10^{2}$ & 21.32 \\
\hline 11.0 & 9.6 & 2564 & 1487 & $7.23 \times 10^{2}$ & 42.00 \\
\hline
\end{tabular}

Table S3. Exchange capacity for isotherm study at the different initial U concentration from $10 \mathrm{ppm}$ to 603 ppm by using basic solution to adjust $\mathrm{pH}$ of exchanged initial solution to the range of 4 to 7 .

\begin{tabular}{|c|c|c|c|c|}
\hline \multirow[t]{2}{*}{ Initial $\mathrm{pH}$} & \multirow[t]{2}{*}{ Final $\mathrm{pH}$} & \multicolumn{2}{|c|}{ U concentration (ppm) } & \multirow[t]{2}{*}{$q(\mathrm{mg} / \mathrm{g})$} \\
\hline & & $C_{0}$ & $C_{f}$ & \\
\hline 6.8 & 6.9 & 10.88 & 5.85 & 5.03 \\
\hline 4.4 & 6.4 & 99.87 & 36.14 & 63.72 \\
\hline 5.1 & 5.6 & 223.11 & 54.92 & 168.18 \\
\hline 4.0 & 4.2 & 341.45 & 85.60 & 255.85 \\
\hline 4.1 & 4.5 & 531.56 & 194.17 & 337.39 \\
\hline 4.1 & 4.1 & 602.89 & 271.88 & 331.01 \\
\hline
\end{tabular}


Table S4. Selected results for the $\mathrm{UO}_{2}{ }^{2+}$-exchange of FJSM-SnS in the presence of a large excess of $\mathrm{Na}^{+}$ ions or $\mathrm{HCO}_{3}{ }^{-}$anion.

\begin{tabular}{|c|c|c|c|c|c|c|c|}
\hline \multirow[t]{2}{*}{ Sample } & \multirow[t]{2}{*}{$\mathrm{pH}$} & \multirow[t]{2}{*}{$\begin{array}{l}\text { Initial } \mathrm{Na} / \mathrm{U} \\
\text { molar ratio }\end{array}$} & \multirow[t]{2}{*}{$\begin{array}{l}\mathrm{V} / \mathrm{m} \\
(\mathrm{mL} / \mathrm{g})\end{array}$} & \multicolumn{2}{|c|}{$\begin{array}{l}\text { U concentration } \\
\qquad(\mathrm{ppb})\end{array}$} & \multirow[t]{2}{*}{$\begin{array}{l}\text { Relative amount of } \\
\text { U removed (\%) }\end{array}$} & \multirow[t]{2}{*}{$K_{\mathrm{d}}(\mathrm{mL} / \mathrm{g})$} \\
\hline & & & & $C_{0}$ & $C_{f}$ & & \\
\hline distilled water $(0.3 \mathrm{M} \mathrm{NaCl})$ & 3.6 & $2.45 \times 10^{4}$ & 1000 & 2915 & 216 & 92.6 & $1.25 \times 10^{4}$ \\
\hline distilled water $\left(0.3 \mathrm{M} \mathrm{NaNO}_{3}\right)$ & 4.8 & $2.41 \times 10^{4}$ & 1000 & 2961 & 151 & 94.9 & $1.86 \times 10^{4}$ \\
\hline distilled water $(0.3 \mathrm{M} \mathrm{NaCl})$ & 3.4 & $2.10 \times 10^{6}$ & 1000 & 34.24 & 0.78 & 97.7 & $4.28 \times 10^{4}$ \\
\hline distilled water (145 ppm $\left.\mathrm{HCO}_{3}^{-}\right)$ & 7.1 & & 1000 & 10.1 & 2.94 & 70.8 & $2.43 \times 10^{3}$ \\
\hline distilled water (145 ppm $\left.\mathrm{HCO}_{3}{ }^{-}\right)$ & 7.4 & & 1000 & 39.7 & 20.6 & 48.2 & $9.29 \times 10^{2}$ \\
\hline distilled water (0.3 $\left.\mathrm{M} \mathrm{NaHCO}_{3}\right)$ & 8.1 & $1.41 \times 10^{6}$ & 1000 & 50.5 & 39.7 & 21.2 & $2.69 \times 10^{2}$ \\
\hline distilled water $\left(0.1 \mathrm{M} \mathrm{NaHCO}_{3}\right)$ & 8.2 & $4.69 \times 10^{5}$ & 1000 & 50.7 & 44.9 & 11.4 & $1.29 \times 10^{2}$ \\
\hline distilled water $\left(0.3 \mathrm{M} \mathrm{NaHCO}_{3}\right)$ & 8.1 & $2.18 \times 10^{4}$ & 1000 & 3784 & 3275 & 13.4 & $1.55 \times 10^{2}$ \\
\hline distilled water $\left(0.1 \mathrm{M} \mathrm{NaHCO}_{3}\right)$ & 8.2 & $6.36 \times 10^{3}$ & 1000 & 3742 & 3108 & 16.9 & $2.04 \times 10^{2}$ \\
\hline
\end{tabular}

Table S5. The results for the $\mathrm{UO}_{2}{ }^{2+}$-exchange of FJSM-SnS with competitive $\mathrm{Ca}^{2+}$ ions.

\begin{tabular}{lllllllll}
\hline $\begin{array}{l}\text { Initial Ca/U } \\
\text { molar ratio }\end{array}$ & $\begin{array}{l}\mathrm{V} / \mathrm{m} \\
(\mathrm{mL} / \mathrm{g})\end{array}$ & $\begin{array}{l}\mathrm{Ca}(\mathrm{ppm}) \\
C_{0}\end{array}$ & $\begin{array}{l}\mathrm{Ca}(\mathrm{ppm}) \\
C_{f}\end{array}$ & $\begin{array}{l}\mathrm{U}(\mathrm{ppb}) \\
C_{0}\end{array}$ & $\begin{array}{l}\mathrm{U}(\mathrm{ppb}) \\
C_{f}\end{array}$ & $\begin{array}{l}\text { Relative } \\
\text { amount of } \mathrm{U}\end{array}$ & $K_{\mathrm{d}} \mathrm{U}(\mathrm{mL} / \mathrm{g})$ & $K_{\mathrm{d}} \mathrm{Ca}(\mathrm{mL} / \mathrm{g})$ \\
\hline $2.89 \times 10^{2}$ & 1000 & 132 & 82 & 2719 & 434 & 84.0 & $5.26 \times 10^{3}$ & $6.03 \times 10^{2}$ \\
$7.45 \times 10^{2}$ & 1000 & 492 & 416 & 3925 & 859 & 78.0 & $3.56 \times 10^{3}$ & $1.83 \times 10^{2}$ \\
$1.19 \times 10^{3}$ & 1000 & 1119 & 1010 & 5557 & 1358 & 75.5 & $3.09 \times 10^{3}$ & $1.07 \times 10^{2}$ \\
$1.62 \times 10^{4}$ & 1000 & 130 & 68 & 47.8 & 14.3 & 70.1 & $2.34 \times 10^{3}$ & $4.73 \times 10^{2}$ \\
$6.05 \times 10^{4}$ & 1000 & 1079 & 836 & 105.9 & 39.8 & 62.4 & $1.66 \times 10^{3}$ & $2.24 \times 10^{2}$ \\
\hline
\end{tabular}

Table S6. $\mathrm{UO}_{2}{ }^{2+}$ ion-exchange results towards trace $\mathrm{UO}_{2}{ }^{2+}$ ions, contaminated seawater and contaminated potable water.

\begin{tabular}{|c|c|c|c|c|c|}
\hline \multirow[t]{2}{*}{ Sample } & \multirow[t]{2}{*}{$\mathrm{pH}$} & \multirow{2}{*}{$\begin{array}{r}\mathrm{V} / \mathrm{m} \\
(\mathrm{mL} / \mathrm{g})\end{array}$} & \multicolumn{2}{|c|}{ U concentration (ppb) } & \multirow{2}{*}{$\begin{array}{l}\text { Relative amount of } \\
\text { U removed }(\%)\end{array}$} \\
\hline & & & $C_{0}$ & $C_{f}$ & \\
\hline distilled water $(0.3 \mathrm{M} \mathrm{NaCl})$ & 6.7 & 100 & 2.07 & very low & close to 100 \\
\hline distilled water $\left(0.15 \mathrm{M} \mathrm{NaNO}_{3}\right)$ & 6.6 & 100 & 1.67 & very low & close to 100 \\
\hline 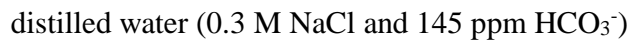 & 7.8 & 100 & 5.60 & 0.37 & 93.3 \\
\hline distilled water $\left(145 \mathrm{ppm} \mathrm{HCO}_{3}^{-}\right)$ & 7.3 & 1000 & 2.59 & 1.89 & 26.9 \\
\hline contaminated seawater & 7.7 & 100 & 32.68 & 29.78 & 8.8 \\
\hline contaminated seawater & 7.7 & 100 & 51.62 & 42.92 & 16.8 \\
\hline contaminated seawater & 7.7 & 100 & 1673 & 1334 & 20.2 \\
\hline contaminated potable water & 7.8 & 100 & 44.15 & 18.06 & 59.0 \\
\hline contaminated potable water & 7.8 & 100 & 13829 & 4340 & 68.6 \\
\hline
\end{tabular}

Natural seawater was from Pacific Ocean located near Sequim Washington. Potable water was found in Evanston, IL. 
Table S7. U removal efficiency of various sorbents in this work and references.

\begin{tabular}{|c|c|c|c|c|c|}
\hline Materials & $\mathrm{pH}$ range & $\begin{array}{c}\mathrm{U} \\
\text { capacity, } \\
q_{\mathrm{m}}(\mathrm{mg} / \mathrm{g})\end{array}$ & $\begin{array}{l}\text { Relative amount } \\
\text { of U removed (\%) }\end{array}$ & $K_{\mathrm{d}}^{\mathrm{U}}(\mathrm{mL} / \mathrm{g})$ & ref \\
\hline FJSM-SnS & $2.1-11$ & 338 & close to 100 & $4.28 \times 10^{4}$ & this work \\
\hline $\mathrm{KMS}-1^{a}$ & $2-10$ & 382 & 99.9 & $1.8 \times 10^{5}$ & Ref 1 \\
\hline Chalcogen-based aerogels ${ }^{b}$ & - & - & $68.1-99.4$ & $(3.1-9.4) \times 10^{4}$ & Ref 2 \\
\hline KTS- $3^{c}$ & $2-12$ & 358 & - & $2.7 \times 10^{4}$ & Ref 3 \\
\hline $\mathrm{S}_{x}-\mathrm{LDH}(x=2,4)^{d}$ & - & 330 & 99.97 & $3.4 \times 10^{6}$ & Ref 4 \\
\hline $\mathrm{Zr}_{6} \mathrm{O}_{4}(\mathrm{OH})_{4} \mathrm{~L}_{6}($ solvates $) \quad(\mathrm{MOF})^{e}$ & $2.5-5$ & 217 & - & - & Ref 5 \\
\hline MOF-76 $f$ & $2.3-6$ & 298 & - & - & Ref 6 \\
\hline $\mathrm{Zn}(\mathrm{HBTC})(\mathrm{L}) \cdot\left(\mathrm{H}_{2} \mathrm{O}\right)_{2}(\mathrm{MOF})^{g}$ & $1-5$ & 115 & 93 & - & Ref 7 \\
\hline multilayer titanate nanotubes & $2-10$ & 333 & - & - & Ref 8 \\
\hline $\mathrm{HSDC}^{h}$ & & 373 & - & - & Ref 9 \\
\hline Magnetic ion-imprinted composite ${ }^{i}$ & $2.0-8.5$ & 354 & $>90$ & - & Ref 10 \\
\hline $\mathrm{ZnO} / \mathrm{CC}^{j}$ & $2-10$ & 431 & close to 100 & - & Ref 11 \\
\hline $\mathrm{Mg}_{2} \mathrm{CO}_{3}(\mathrm{OH})_{2} / \mathrm{CC}^{k}$ & $2-10$ & 370 & - & - & Ref 12 \\
\hline Cs-birnessite & & - & close to 100 & $1.6 \times 10^{6}$ & Ref 13 \\
\hline Li-birnessite & & - & 99.9 & $1.8 \times 10^{5}$ & Ref 13 \\
\hline Na-birnessite & & - & 99.6 & $4.9 \times 10^{4}$ & Ref 13 \\
\hline K-birnessite & & - & 99.8 & $8.8 \times 10^{4}$ & Ref 13 \\
\hline
\end{tabular}

KMS-1 ${ }^{a}$ : layered sulfide $\mathrm{K}_{2} \mathrm{MnSn}_{2} \mathrm{~S}_{6}$ (KMS-1). Chalcogen-based aerogels ${ }^{b}$ : Chalcogels of $\mathrm{Co}_{0.7} \mathrm{Bi}_{0.3} \mathrm{MoS}_{4}, \mathrm{Co}_{0.7} \mathrm{Cr}_{0.3} \mathrm{MoS}_{4}, \mathrm{Co}_{0.5} \mathrm{Ni}_{0.5} \mathrm{MoS}_{4}$, $\mathrm{PtGe}_{2} \mathrm{~S}_{5}$, and $\mathrm{Sn}_{2} \mathrm{~S}_{3}$. KTS-3 ${ }^{c}$ : layered sulfide $\mathrm{K}_{2 x} \mathrm{Sn}_{4-x} \mathrm{~S}_{8-x}(x=0.65-1)$. $\mathrm{S}_{x}-\mathrm{LDH}(x=2,4)^{d}$ : polysulfide/LDH composites (LDH is $\mathrm{Mg} / \mathrm{Al}$ layered double hydroxide). $\quad \mathrm{L}$ in $\mathrm{Zr}_{6} \mathrm{O}_{4}(\mathrm{OH})_{4} \mathrm{~L}_{6}$ (solvates) $\quad(\mathrm{MOF})^{e}$ : TPDC bridging ligands containing orthogonal phosphorylurea groups (TPDC is p,p'-terphenyldicarboxylic acid). MOF-76: MOF constructed with lanthanide ion and 1,3,5-benzenetricarboxylate (BTC) ligand. In $\mathrm{Zn}(\mathrm{HBTC})(\mathrm{L}) \cdot\left(\mathrm{H}_{2} \mathrm{O}\right)_{2}(\mathrm{MOF})^{g}, \mathrm{H}_{3} \mathrm{BTC}=1,3,5$-benzenetricarboxylic acid, $\mathrm{L}=N^{4}, N^{4}$-di(pyridine-4-yl)biphenyl-4,4'-dicarboxamide. $\mathrm{HSDC}^{h}$ : polyphenolic-hydroxyl functionalized material. Magnetic ion-imprinted composite ${ }^{i} \mathrm{Fe}_{3} \mathrm{O}_{4}$ magnetic microspheres coated with a thin $\mathrm{SiO}_{2}$ film $\left(\mathrm{Fe}_{3} \mathrm{O}_{4} / \mathrm{SiO}_{2}\right)$. $\mathrm{ZnO} / \mathrm{CC}^{j}$ : $\mathrm{ZnO}$ nanorod arrays on cotton cloth. $\mathrm{Mg}_{2} \mathrm{CO}_{3}(\mathrm{OH})_{2} / \mathrm{CC}^{k}$ : Magnesium carbonate basic coating on cotton cloth. 


\section{The theoretical ion-exchange capacity:}

The theoretical ion-exchange capacity means $U$ adsorption capacity of $\left(\mathrm{Me}_{2} \mathrm{NH}_{2}\right)_{1.33}\left(\mathrm{Me}_{3} \mathrm{NH}\right)_{0.67} \mathrm{Sn}_{3} \mathrm{~S}_{7} \cdot 1.25 \mathrm{H}_{2} \mathrm{O}$ (FJSM-SnS) when all organic amine cations in FJSM-SnS are completely exchanged by uranyl. According to molecule formula of FJSM-SnS, theoretically per molecule of FJSM-SnS can capture one $\mathrm{UO}_{2}{ }^{2+}$ ions. Here, the theoretical ion-exchange capacity $q\left(\mathrm{mg} \cdot \mathrm{g}^{-1}\right)$ for $\mathrm{U}$ of FJSM-SnS can be calculated from the equation $\mathrm{S} 1$.

$$
\begin{gathered}
q(\text { theoretical })=\frac{\text { Atomic weight of uranium }}{\text { Formula weight of } \mathrm{FJSM}-\mathrm{SnS}} \times 1000=\frac{238.03}{704.54} \times 1000 \\
=337.85 \mathrm{mg} \cdot \mathrm{g}^{-1} \quad \text { (Equation S1) }
\end{gathered}
$$

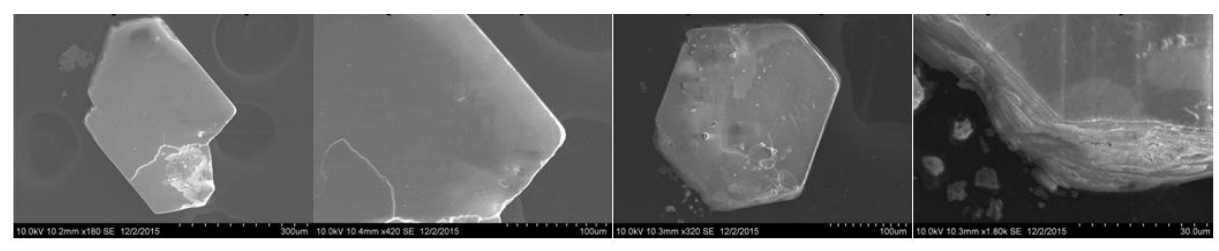
S1-1
S1-2
S1-3
S1-4

Figure S1. SEM images of pristine FJSM-SnS crystals (S1-1 and S1-2) and $\mathrm{UO}_{2}{ }^{2+}$-exchanged products (S1-3 and S1-4).

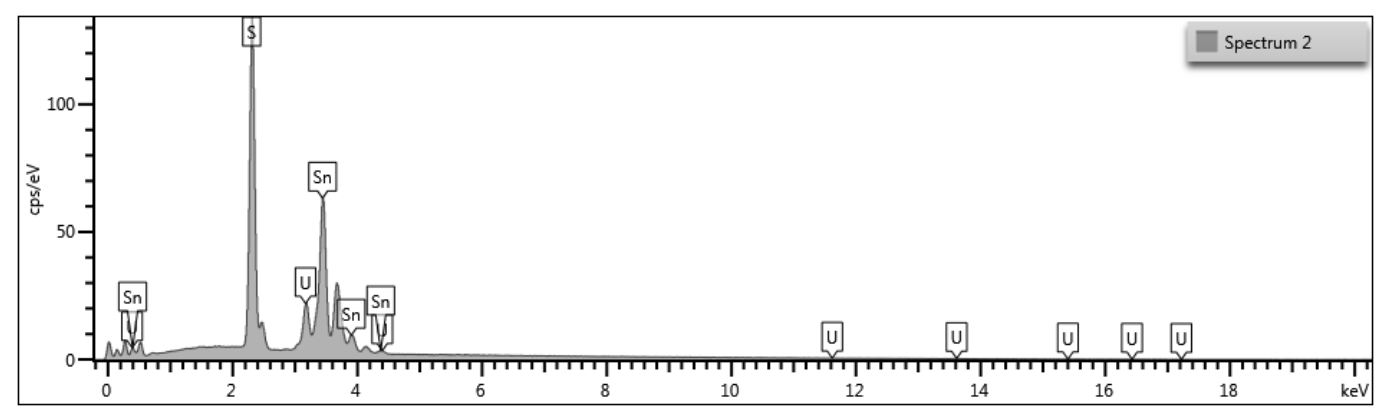

\begin{tabular}{|l|l|}
\hline Element & Atomic \% \\
\hline S & 63.63 \\
\hline Sn & 30.29 \\
\hline U & 6.09 \\
\hline Total: & 100 \\
\hline
\end{tabular}

Figure S2. EDS analysis results of $\mathrm{UO}_{2}{ }^{2+}$-exchanged FJSM-SnS products. 


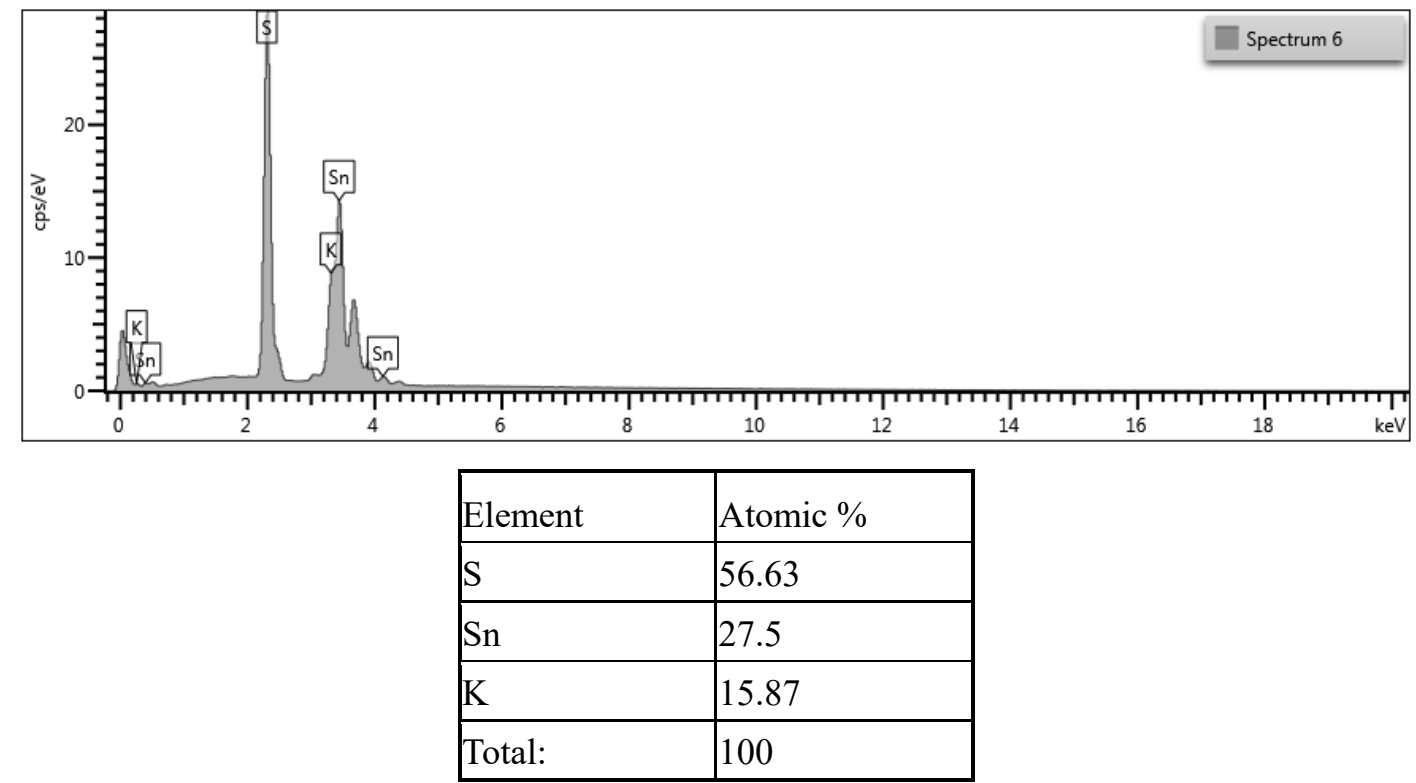

Figure S3. EDS analysis results of the eluted product with the near composition of $\mathrm{KSn}_{1.73} \mathrm{~S}_{3.56}$.

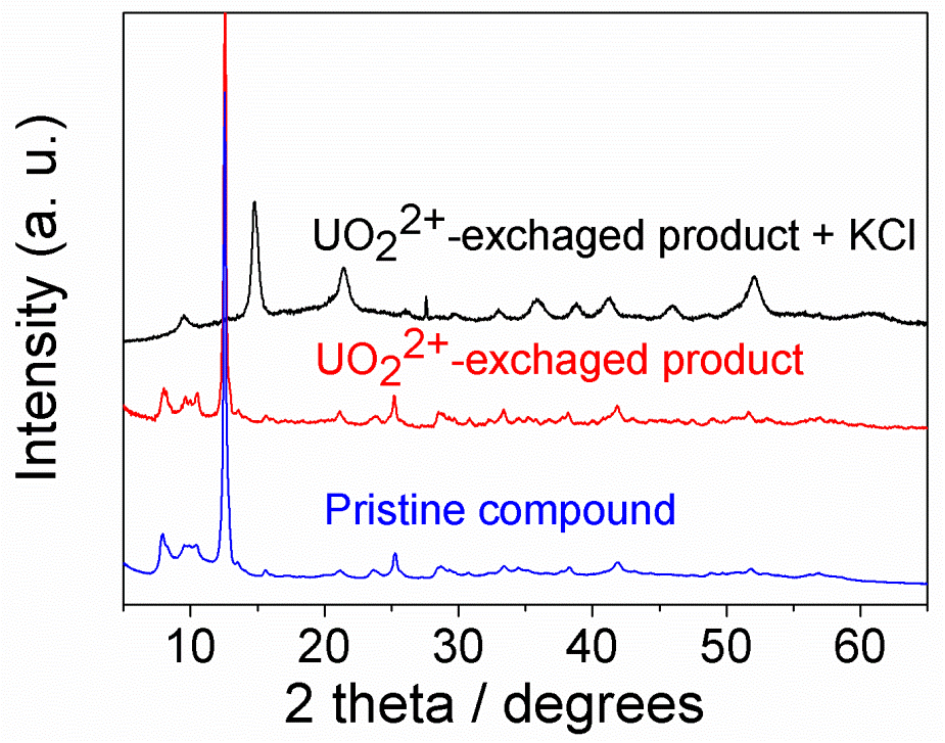

Figure S4. PXRD patterns of eluted products, $\mathrm{UO}_{2}{ }^{2+}$-exchanged products and the pristine compound. 


\section{REFERENCES}

1. Manos, M. J.; Kanatzidis, M. G. J. Am. Chem. Soc. 2012, 134, 16441-16446.

2. Riley, B. J.; Chun, J.; Um, W.; Lepry, W. C.; Matyas, J.; Olszta, M. J.; Li, X. H.; Polychronopoulou, K.; Kanatzidis, M. G. Environ. Sci. Technol. 2013, 47, 7540-7547.

3. Sarma, D.; Malliakas, C. D.; Subrahmanyam, K. S.; Islama, S. M.; Kanatzidis, M. G. Chem. Sci. 2016, 7, 1121-1132.

4. Ma, S. L.; Huang, L.; Ma, L. J.; Shim, Y.; Islam, S. M.; Wang, P. L.; Zhao, L. D.; Wang, S. C.; Sun, G. B.; Yang, X. J.; Kanatzidis, M. G. J. Am. Chem. Soc. 2015, 137, 3670-3677.

5. Carboni, M.; Abney, C. W.; Liu, S. B.; Lin, W. B. Chem. Sci. 2013, 4, 2396-2402.

6. Yang, W. T.; Bai, Z. Q.; Shi, W. Q.; Yuan, L. Y.; Tian, T.; Chai, Z. F.; Wang, H.; Sun, Z. M. Chem. Commun. 2013, 49, 10415-10417.

7. Wang, L. L.; Luo, F.; Dang, L. L.; Li, J. Q.; Wu, X. L.; Liu, S. J.; Luo, M. B. J. Mater. Chem. A 2015, 3, 13724-13730.

8. Liu, W.; Zhao, X.; Wang, T.; Zhao, D. Y.; Ni, J. R. Chem. Eng. J. 2016, 286, 427-435.

9. Liu, J.; Li, J.; Yang, X. D.; Song, Q.; Bai, C. Y.; Shi, Y.; Zhang, L.; Liu, C. X.; Li, S. J.; Ma, L. J. Mater. Lett. 2013, 97, 177-180.

10. Liu, M. C.; Chen, C. L.; Wen, T.; Wang, X. K. Dalton Trans. 2014, 43, 7050-7056.

11. Zhang, L.; Zhang, L.; Wu, T. H.; Jing, X. Y.; Li, R. M.; Liu, J. Y.; Liu, Q.; Wang, J. RSC Adv. 2015, 5, 53433-53440.

12. Zhang, L.; Jing, X. Y.; Li, R. M.; Liu, Q.; Liu, J. Y.; Zhang, H. S.; Hu, S. X.; Wang, J. RSC Adv. 2015, 5, 23144-23151.

13. Al-Attar, L.; Dyer, A. J. Mater. Chem. 2002, 12, 1381-1386. 